\title{
Potentially Inappropriate Opioid Prescribing, Overdose, and Mortality in Massachusetts, 2011-2015
}

\author{
Adam J. Rose, MD MSc FACP ${ }^{1,2}$, Dana Bernson, MPH ${ }^{3}$, Kenneth Kwan Ho Chui, PhD MPH', \\ Thomas Land, PhD ${ }^{3}$, Alexander Y. Walley, MD MSc ${ }^{2,3}$, Marc R. LaRochelle, MD MPH', \\ Bradley D. Stein, MD PhD ${ }^{5,6}$, and Thomas J. Stopka, PhD MHS $S^{4,7}$
}

IRAND Corporation, Boston, MA, USA; '2Section of General Internal Medicine, Boston University School of Medicine and Boston Medical Center, Boston, MA, USA; ${ }^{3}$ Massachusetts Department of Public Health, Boston, MA, USA; ${ }^{4}$ Tufts University School of Medicine, Boston, MA, USA; ${ }^{5}$ RAND Corporation, Pittsburgh, PA, USA; UUniversity of Pittsburgh School of Medicine, Pittsburgh, PA, USA; ${ }^{7}$ Tufts Clinical and Translational Sciences Institute, Boston, MA, USA.

BACKGROUND: Potentially inappropriate prescribing (PIP) may contribute to opioid overdose.

OBJECTIVE: To examine the association between PIP and adverse events.

DESIGN: Cohort study.

PARTICIPANTS: Three million seventy-eight thousand thirty-four individuals age $\geq 18$, without disseminated cancer, who received prescription opioids between 2011 and 2015.

MAIN MEASURES: We defined PIP as (a) morphine equivalent dose $\geq 100 \mathrm{mg} /$ day in $\geq 3$ months; (b) overlapping opioid and benzodiazepine prescriptions in $\geq 3$ months; (c) $\geq 4$ opioid prescribers in any quarter; (d) $\geq 4$ opioiddispensing pharmacies in any quarter; (e) cash purchase of prescription opioids on $\geq 3$ occasions; and (f) receipt of opioids in 3 consecutive months without a documented pain diagnosis. We used Cox proportional hazards models to identify PIP practices associated with non-fatal opioid overdose, fatal opioid overdose, and all-cause mortality, controlling for covariates.

KEY RESULTS: All six types of PIP were associated with higher adjusted hazard for all-cause mortality, four of six with non-fatal overdose, and five of six with fatal overdose. Lacking a documented pain diagnosis was associated with non-fatal overdose (adjusted hazard ratio [AHR] 2.21, 95\% confidence interval [CI] 2.02-2.41), as was high-dose opioids (AHR 1.68, 95\% CI 1.59-1.76). Co-prescription of benzodiazepines was associated with fatal overdose (AHR 4.23, 95\% CI 3.85-4.65). High-dose opioids were associated with all-cause mortality (AHR 2.18, 95\% CI 2.14-2.23), as was lacking a documented pain diagnosis (AHR 2.05, 95\% CI 2.01-2.09). Compared to those who received opioids without PIP, the hazard for fatal opioid overdose with one, two, three, and $\geq$ four PIP subtypes were 4.24, 7.05, 10.28, and 12.99 (test of linear trend, $p<0.001$ ).

Electronic supplementary material The online version of this article (https://doi.org/10.1007/s11606-018-4532-5) contains supplementary material, which is available to authorized users.

Received May 8, 2018

Revised June 5, 2018

Accepted June 5, 2018

Published online June 14, 2018
CONCLUSIONS: PIP was associated with higher hazard for all-cause mortality, fatal overdose, and non-fatal overdose. Our study implies the possibility of creating a risk score incorporating multiple PIP subtypes, which could be displayed to prescribers in real time.

KEY WORDS: potentially inappropriate prescribing; overdose; opioids; mortality.

J Gen Intern Med 33(9):1512-9

DOI: $10.1007 / \mathrm{s} 11606-018-4532-5$

(c) Society of General Internal Medicine 2018

\section{INTRODUCTION}

Opioid overdose is a public health crisis. Most individuals who misuse opioids are initially exposed to prescription opioids. ${ }^{2} 3$ Therefore, improving opioid prescribing may reduce the risk of opioid misuse and overdose death. ${ }^{2}$ Recently, attention has focused on potentially inappropriate prescribing of opioids (PIP) as a risk factor for fatal overdose, given its frequency and growth over time. ${ }^{4}$ Studies have shown that high-dose opioids, defined as $\geq 100 \mathrm{mg}$ morphine equivalents (MMEs) per day, or co-prescription of opioids with benzodiazepines, are associated with opioid overdose. ${ }^{7-17}$ Other studies have shown that multiple prescribers or multiple pharmacies are associated with opioid overdose. ${ }^{7}, 14,18$ Using a uniquely powerful database created by the Massachusetts State legislature to address the opioid crisis, we examined the prevalence and consequences of PIP in Massachusetts.

\section{METHODS}

\section{Database}

Chapter 55 of the Acts of 2015 ("Chapter 55") mandated that the Massachusetts Department of Public Health facilitates the analysis of data from multiple Massachusetts government agencies during May and June of 2017. Data included the state's prescription monitoring program, all-payer claims database (APCD), mortality including autopsy and postmortem toxicology, demographics and area-level socioeconomic 
status, ambulance records/emergency medical services, and acute hospital case mix, and contained information on more than $98 \%$ of Massachusetts' population. ${ }^{19}, 20$ The online state prescription monitoring program includes all controlled substances dispensed by Massachusetts pharmacies or delivered to a Massachusetts resident by mail. Federal facility pharmacies are not included. While pharmacists are encouraged to register and use the online state prescription monitoring program, which facilitates monitoring of prescriptions from all prescribers and pharmacies, use is not mandatory. However, prescribers are required to access the online state prescription monitoring program prior to prescribing opioids or benzodiazepines.

Chapter 55 data quality and completeness are described in detail in the report delivered to the Massachusetts Legislature by the Massachusetts Department of Public Health, especially in Online Appendix A of that report. ${ }^{21}$ This study was exempted from review by the Institutional Review Boards of the RAND Corporation and the Massachusetts Department of Public Health.

\section{Cohort}

We used state prescription monitoring program data to identify all patients age $\geq 18$ who filled at least one opioid prescription $(n=3,078,034)$ during the five-year study period (2011-2015). We excluded individuals with regionally or distantly disseminated cancer (defined in Online Appendix A), and individuals receiving buprenorphine or methadone for opioid use disorder detoxification or treatment. The flow diagram depicting these exclusions, and the final sample, is in Figure 1.

Patients were only considered to leave the cohort by dying; while some may have moved out of state, it is not possible to distinguish this from a period during which they consumed no healthcare (and thus would not appear in the APCD). A table with the number of patients who entered and left the cohort each year is in Online Appendix B.

\section{Potentially Inappropriate Prescribing}

We identified patients receiving PIP. The first four of our PIP variables have been used in previous studies, ${ }^{7}-18$ while the last two have not previously been used in a similar study.

1) High-dose opioids. We calculated the daily dose equivalent for each patient in each month in MME by summing the MMEs for all prescriptions over a month and dividing by the number of days in that month covered by the prescriptions (Online Appendix C). We considered a patient to have received high-dose opioids if they had an average MME $\geq 100 \mathrm{mg}$ /day in three separate (but not necessarily consecutive) months, at any time during the five-year study.

2) Co-prescribing of opioids and benzodiazepines. We defined a patient as having received overlapping

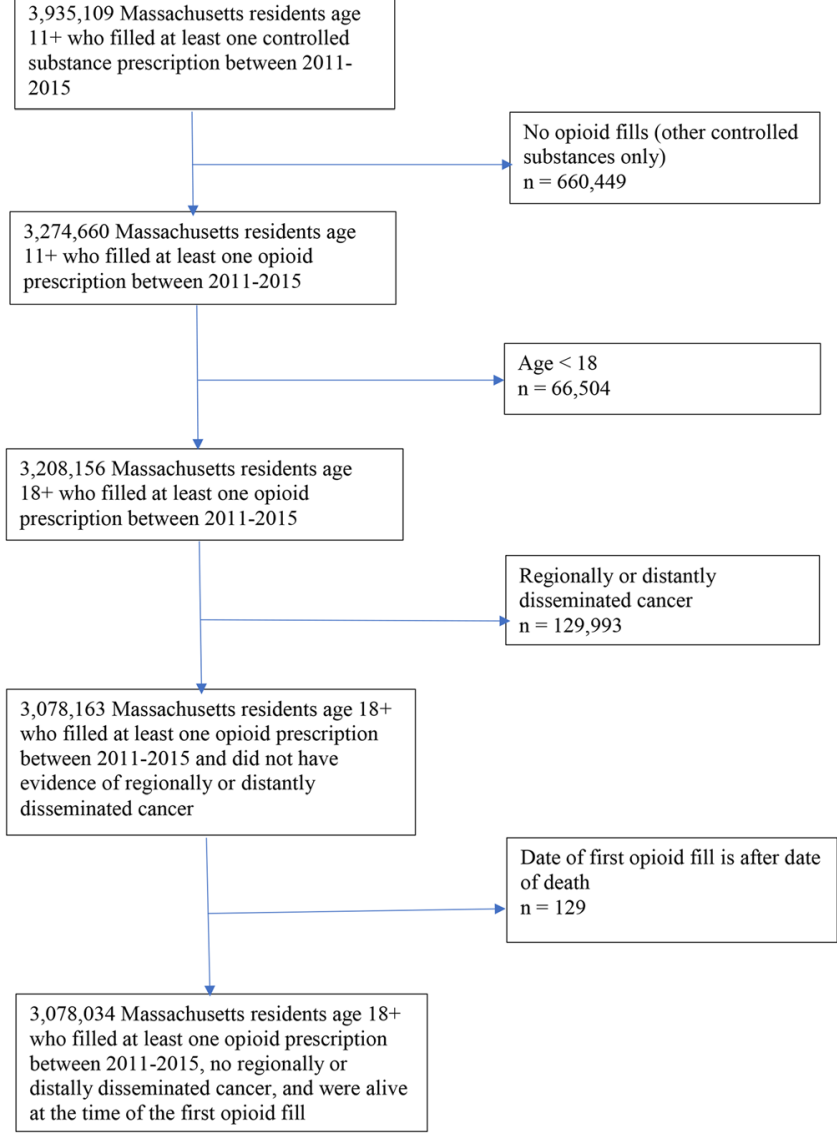

Figure 1 Flow diagram of exclusions and final study cohort.

prescriptions when the patient had received opioid and benzodiazepine prescriptions that overlapped by at least one day in three separate (but not necessarily consecutive) months, at any time during the five-year study.

3) Multiple opioid prescribers. We defined patients as having multiple prescribers if they had $\geq 4$ opioid prescribers in any quarter of a calendar year.

4) Multiple opioid pharmacies. We considered patients to have multiple pharmacies if they received opioids from $\geq 4$ pharmacies in any quarter.

5) Cash purchases of prescription opioids. It was considered PIP if the patient made three or more cash purchases during any quarter between 2011 and 2015.

6) Lack of a documented pain diagnosis. We defined it as PIP if a patient received opioids for three consecutive months without recording an ICD-9 or ICD-10 code claim for a pain diagnosis at any point during the study period (as defined in Online Appendix D).

We characterized each patient as meeting criteria for none of the six kinds of PIP, one, two, three, or four or more.

\section{Patient Outcomes}

Outcomes included (1) non-fatal opioid overdose; (2) fatal opioid overdose; and (3) all-cause mortality. Non-fatal overdose was defined in emergency medical services data using the 
Massachusetts Department of Public Health algorithm and in the case mix data using ICD-9 or ICD-10 codes (Online Appendix E). For non-fatal overdose, we considered only the first occurrence. Fatal overdose and all-cause mortality were defined using state mortality data (Online Appendix F). All-cause mortality was included given opioids' potential contribution to death both through unrecognized opioid over$\operatorname{doses}^{22,23}$ and through auto accidents, falls, and other trauma to which opioid use contributed.

\section{Covariates}

Available covariates, including demographics and comorbid conditions, were selected due to likely association with PIP, overdose, or mortality. Individuals were categorized into age groups in 10-year intervals $(18-29,30-39, \ldots, 70-79$, and $80+)$ and categorized as male or female. We did not characterize race/ethnicity because data were missing in nearly half the cases. Using 2011-2015 American Community Survey data, ${ }^{24}$ we characterized community-level socioeconomic status (SES) by the percentage of households below the federal poverty level in the patient's zip code of residence. ${ }^{25}$ Major depression and alcohol use disorder were identified by at least one ICD-9 or ICD-10 code during the study period (Online Appendices $\mathrm{G}$ and $\mathrm{H}$ ), and we controlled for all other comorbidities using the Elixhauser comorbidity score, ${ }^{26}$ a widely used all-purpose comorbidity score based on ICD codes. Residence in a rural municipality was identified using the Massachusetts Department of Public Health's Rural Health Office definition. Individuals with a history of incarceration in either prison or jail during the study period were identified using Department of Corrections and Department of Justice data, and individuals with a history of homelessness during the study period were identified using data from multiple sources, as discussed in Online Appendix I. All covariates were modeled as categorical variables, except the Elixhauser score, which was continuous.

Individuals in our sample who were missing information needed to characterize area-level poverty $(1.5 \%)$ and rural municipality $(0.8 \%)$ were assigned "missing" for these variables, allowing those individuals to enter into the regression analyses.

\section{Statistical Analyses}

We examined the Pearson correlation among the six PIP subtypes. We used Cox proportional hazard models to examine the hazard for each of our three outcomes, controlling for covariates. The independent variables were each PIP subtype, structured as binary variables (ever/never). We examined the association of six PIP subtypes with each outcome-first alone, and then together with the other PIP subtypes in a single model. We also examined the association between the number of PIP subtypes to which each patient had been exposed (between 0 and 4+ PIP subtypes) and each outcome. For non-fatal overdoses, PIP exposure was only counted if it occurred before the overdose event; we did not assess recency of PIP exposure.

We did not model PIP exposure as a time-varying covariate, given that in some cases adverse outcomes may lag PIP exposure by months or years. We therefore chose to model PIP exposure as a binary variable, although it should be kept in mind that our PIP variables required repeated exposures to meet the threshold definition of having received PIP.

As with any observational study, ours could be influenced by unresolved confounding. As a sensitivity analysis, we calculated the $E$-value, ${ }^{27}$ a measure of how strongly associated an unobserved confounder would need to be with both our independent and dependent variables to completely explain our results (i.e., reduce the estimate to the null value). All analyses were performed using SAS Studio, version 3.5 (SAS Corporation, Cary, NC).

\section{RESULTS}

Three million seventy-eight thousand thirty-four adult Massachusetts residents-about $57 \%$ of the state's adult population - received at least one opioid prescription between 2011 and 2015. The number of adults receiving an opioid prescription remained stable over time - about 1.13 to 1.16 million adults in each study year. Prescription opioid recipients varied widely by age (Table 1); 55\% were female. Almost onefifth had an insurance claim for major depression (17.7\%); $4.6 \%$ had a claim for alcohol use disorder. The mean Elixhauser comorbidity index for the sample was 2.11 (prevalence of Elixhauser comorbidities in Online Appendix J). Among all who received an opioid prescription, 4.3\% died before the end of the study, including $0.11 \%$ who experienced fatal opioid overdose; $0.72 \%$ experienced non-fatal opioid overdose. The mean person-time under observation was 1105 days; the median was 1199 days.

Many patients received potentially inappropriate prescriptions just once, and a subset received them repeatedly (Table 2). We generally required at least 3 months of such exposure to constitute PIP for the purposes of this study. Based on our definitions, $88.6 \%$ of recipients of prescription opioids did not have any PIP, $6.9 \%$ had one type, $2.7 \%$ had two, $1.2 \%$ had three, and $0.6 \%$ had four or more types of PIP. The annual prevalence of PIP increased from 2011 to 2012 (from 168,781 to 187,888) and then trended down to 162,575 in 2015 . Coprescription of opioids and benzodiazepines was the most common type of PIP and rose consistently from 57,878 in 2011 to 64,208 in 2015; all other PIP variables steadily decreased (Online Appendix K).

PIP was more common with increasing age: $13.5 \%$ of patients who experienced PIP were age $80+$, while only $7.6 \%$ of opioid recipients who did not experience PIP were age $80+$ (Table 1). PIP was slightly more common among females, and markedly more common among those with 
Table 1 Comparison of Characteristics and Clinical Outcomes Between Patients Receiving Any PIP vs. No PIP, Among Massachusetts Adults Who Received Prescribed Opioids at Least Once Between 2011 and $2015(n=3,078,034)$

\begin{tabular}{|c|c|c|c|}
\hline Variable & $\begin{array}{l}\text { All recipients of } \\
\text { prescription opioids } \\
(n=\mathbf{3 , 0 7 8 , 0 3 4 )}\end{array}$ & $\begin{array}{l}\text { No PIP } \\
(n=2,725,838)\end{array}$ & $\begin{array}{l}\text { Any PIP } \\
(n=352,325)\end{array}$ \\
\hline \multicolumn{4}{|l|}{ Age } \\
\hline $18-29$ & $528,860(17.2 \%)$ & $514,295(18.9 \%)$ & $14,565(4.1 \%)$ \\
\hline $30-39$ & $477,858(15.5 \%)$ & $440,320(16.2 \%)$ & $37,538(10.6 \%)$ \\
\hline $40-49$ & $480,128(15.6 \%)$ & $427,807(15.7 \%)$ & $52,321(14.8 \%)$ \\
\hline $50-59$ & $578,270(18.8 \%)$ & $495,326(18.2 \%)$ & $82,944(23.5 \%)$ \\
\hline $60-69$ & $478,256(15.5 \%)$ & $407,803(15.0 \%)$ & $70,453(20.0 \%)$ \\
\hline $70-79$ & $270,414(8.6 \%)$ & $226,185(8.3 \%)$ & $44,229(12.6 \%)$ \\
\hline $80+$ & $264,248(8.6 \%)$ & $213,981(7.8 \%)$ & $50,267(14.3 \%)$ \\
\hline \multicolumn{4}{|r|}{ 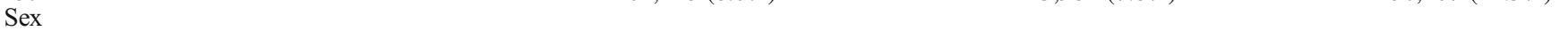 } \\
\hline Male & $1,383,600(45.0 \%)$ & $1,238,411(45.4 \%)$ & $145,189(41.2 \%)$ \\
\hline Female & $1,694,430(55.0 \%)$ & $1,487,302(54.6 \%)$ & $207,128(58.8 \%)$ \\
\hline \multicolumn{4}{|l|}{ Zip code level percentage of } \\
\hline \multicolumn{4}{|l|}{ households below federal poverty } \\
\hline $0-9.99 \%$ & $1,586,783(51.6 \%)$ & $1,407,490(51.6 \%)$ & $179,293(50.9 \%)$ \\
\hline $10-19.99 \%$ & $878,677(28.6 \%)$ & $775,039(28.4 \%)$ & $103,638(29.4 \%)$ \\
\hline $20-29.99 \%$ & $414,883(13.5 \%)$ & $365,278(13.4 \%)$ & $49,605(14.1 \%)$ \\
\hline $30 \%+$ & $151,096(4.9 \%)$ & $135,557(5.0 \%)$ & $15,539(4.4 \%)$ \\
\hline Missing & $46,595(1.5 \%)$ & $42,353(1.6 \%)$ & $4242(1.2 \%)$ \\
\hline \multicolumn{4}{|l|}{ Rural town or city of residence } \\
\hline Non-rural & $2,729,278(89.4 \%)$ & $2,417,319(89.4 \%)$ & $311,959(89.0 \%)$ \\
\hline Rural & $324,526(10.6 \%)$ & $285,837(10.6 \%)$ & $38,689(11.0 \%)$ \\
\hline Missing & $24,230(0.8 \%)$ & $22,561(0.8 \%)$ & $1669(0.5 \%)$ \\
\hline History of incarceration & $32,093(1.0 \%)$ & $24,810(0.9 \%)$ & $7283(2.1 \%)$ \\
\hline History of homelessness & $27,370(0.9 \%)$ & $19,512(0.3 \%)$ & $7858(2.2 \%)$ \\
\hline \multicolumn{4}{|l|}{ Specific comorbid conditions } \\
\hline Major depression & $544,149(17.7 \%)$ & $419,869(15.4 \%)$ & $124,280(35.3 \%)$ \\
\hline Alcohol use disorder & $140,200(4.6 \%)$ & $106,734(3.9 \%)$ & $33,466(9.5 \%)$ \\
\hline Mean Elixhauser comorbidity score (SD) & $2.11(2.87)$ & $3.85(4.00)$ & $1.89(2.61)$ \\
\hline
\end{tabular}

PIP potentially inappropriate opioid prescribing

major depression and alcohol use disorder. All six PIP variables were correlated with each other (Online Appendix L), meaning that a patient who experienced one type of PIP was also more likely to experience other types of PIP ( $p<0.001$ for all correlations). The strongest correlation was between $4+$ prescribers and $4+$ pharmacies $(r=$ $0.34)$, followed by $\geq 100$ MMEs with opioid + benzodiazepines $(r=0.32)$. All other correlations were below 0.3 .

All six kinds of PIP were associated with all-cause mortality in the fully adjusted model (Table 3; full results of all models in Online Appendices M,N, and O). The strongest associations for all-cause mortality were with receipt of high-dose opioids (adjusted hazard ratio [AHR] 2.18, 95\% confidence interval [CI 2.14-2.23) and lacking a documented pain diagnosis (AHR 2.05, 95\% CI 2.01-2.09). Five of the six kinds of PIP were associated with fatal opioid overdose-the exception being cash payments. The strongest association for fatal overdose was with benzodiazepine co-prescription (AHR 4.23, 95\% CI 3.85-4.65). Four kinds of PIP were associated with non-fatal overdose, most prominently lacking a documented pain diagnosis (AHR 2.21, 95\% CI 2.02-2.41) and high-dose opioids (AHR 1.68, 95\% CI 1.59-1.76). We also examined the association with the cumulative number of PIP subtypes and found increasing hazard for adverse events as the number of PIP types increased (Table 4). A table of $E$-values, a measure of the potential for unobserved confounding to fully explain each estimate, is given in Online Appendix P.

\section{DISCUSSION}

We examined the association of PIP with all-cause mortality, fatal opioid overdose, and non-fatal opioid overdose among adult Massachusetts residents without cancer from 2011 to 2015. More than 1 in 10 (11.4\%) recipients of prescription opioids experienced at least one kind of PIP. All six kinds of PIP were associated with higher hazard for death, all but one with fatal opioid overdose, and all but two with non-fatal overdose. We found that patients had markedly increased hazard for adverse events as they were exposed to a higher number of PIP subtypes.

We found that PIP became more common with increasing age, a finding at odds with the public image of the opioid crisis as a problem of young people. It may be that older adults simply receive more medications, leading to issues such as co-prescribing of benzodiazepines, or that prescribers are simply more conservative when prescribing to younger patients, because of a perception that they are more likely to misuse opioid analgesics. This finding will be an important topic for future study.

We compared our estimates regarding the prevalence of PIP to several relatively recent studies. A report by the Centers for Disease Control using data from eight states in 2013 (the midpoint of our study period) found that the proportion of patients receiving $\geq 100$ MMEs ranged from 
Table 2 Frequencies and Percentages of Potentially Inappropriate Opioid Prescribing (PIP) in Massachusetts, 2011-2015 $(n=3,078,034)$

\begin{tabular}{|c|c|c|}
\hline Variable & Frequency & Percent \\
\hline \multicolumn{3}{|l|}{$\begin{array}{l}\text { Number of months with } \geq 100 \mathrm{mg} \\
\text { of morphine equivalents }\end{array}$} \\
\hline None & $2,847,155$ & 92.5 \\
\hline 1 & 122,104 & 4.0 \\
\hline 2 & 33,608 & 1.1 \\
\hline 3 or more & 75,167 & 2.4 \\
\hline \multicolumn{3}{|l|}{$\begin{array}{l}\text { Number of months co-prescribed } \\
\text { benzodiazepines and opioids }\end{array}$} \\
\hline None & $2,484,141$ & 80.7 \\
\hline 1 & 326,047 & 10.6 \\
\hline 2 & 85,274 & 2.8 \\
\hline 3 or more & 182,572 & 5.9 \\
\hline \multicolumn{3}{|l|}{$\begin{array}{l}\text { Highest number of opioid } \\
\text { prescribers per quarter }\end{array}$} \\
\hline 1 & $2,224,622$ & 73.3 \\
\hline 2 & 528,324 & 17.4 \\
\hline 3 & 176,788 & 5.8 \\
\hline $4+$ & 104,464 & 3.4 \\
\hline \multicolumn{3}{|l|}{$\begin{array}{l}\text { Highest number of opioid } \\
\text { pharmacies per quarter }\end{array}$} \\
\hline 1 & $2,485,483$ & 81.9 \\
\hline 2 & 436,547 & 14.4 \\
\hline 3 & 82,952 & 2.7 \\
\hline $4+$ & 29,216 & 1.0 \\
\hline \multicolumn{3}{|l|}{$\begin{array}{l}\text { Highest number of cash } \\
\text { payments for opioids in a quarter }\end{array}$} \\
\hline None & $2,289,309$ & 74.4 \\
\hline 1 & 557,860 & 18.1 \\
\hline 2 & 132,372 & 4.3 \\
\hline 3 or more & 98,493 & 3.2 \\
\hline \multicolumn{3}{|l|}{$\begin{array}{l}\text { Duration of therapy by lack } \\
\text { of documented pain diagnosis }\end{array}$} \\
\hline $\begin{array}{l}\text { Less than } 3 \text { months/with documented pain } \\
\text { diagnosis }\end{array}$ & $1,550,643$ & 50.4 \\
\hline $\begin{array}{l}\text { Less than } 3 \text { months/no documented pain } \\
\text { diagnosis }\end{array}$ & $1,152,989$ & 37.5 \\
\hline $\begin{array}{l}3 \text { or more months/with documented pain } \\
\text { diagnosis }\end{array}$ & 296,951 & 9.6 \\
\hline $\begin{array}{l}3 \text { or more months/no documented pain } \\
\text { diagnosis }\end{array}$ & 77,451 & 2.5 \\
\hline \multicolumn{3}{|l|}{$\begin{array}{l}\text { Number of PIP subtypes received } \\
\text { (out of a possible } 6)^{*}\end{array}$} \\
\hline None & $2,725,717$ & 88.6 \\
\hline 1 & 213,671 & 6.9 \\
\hline 2 & 84,437 & 2.7 \\
\hline 4 & 36,147 & 1.2 \\
\hline 4,5 , or 6 & 18,062 & 0.6 \\
\hline
\end{tabular}

Definitions for the six types of potentially inappropriate opioid prescribing (PIP): (1) more than $100 \mathrm{mg}$ morphine equivalents on three or more occasions; (2) benzodiazepine/opioid overlap on three or more occasions; (3) four or more prescribers in any one quarter; (4) four or more pharmacies in any one quarter; (5) three or more cash payments; and (6) three consecutive months with opioids despite lack of a documented pain diagnosis. The definitions of the six PIP variables are in italics

PIP potentially inappropriate opioid prescribing

*The "number of PIP criteria fulfilled" variable in this table represents all exposures to PIP while the patient was alive, which was the independent variable for models of all-cause mortality and fatal opioid overdose. A separate version of the PIP count variable was also created for use with regression analyses for non-fatal opioid overdose, which only counts PIP exposures prior to the first non-fatal overdose event

8.1 to $16 \%$ across states. ${ }^{5}$ By comparison, we found that $7.5 \%$ of patients had a high daily dose on at least one occasion, suggesting that high-dose opioids may be less common in Massachusetts than other states. In an analysis of the MarketScan Commercial Claims database from
2009 , opioid-benzodiazepine overlap was seen in $2.2 \%$ of opioid recipients on one occasion and $5.8 \%$ on two or more occasions, ${ }^{4}$ compared to 10.6 and $8.7 \%$ in our study. The higher rates in our study may be attributable to the inclusion of non-commercially insured populations.

We also compared our study to several recent studies that have also examined potential links between PIP and opioid overdose (Online Appendix Q). Our findings were generally similar to the previous studies, although we did examine some new PIP variables not previously included. Our study is also distinguished from the others in terms of the outcomes we used. We included allcause mortality as an outcome because it is not subject to ascertainment bias. Because recognition of fatal overdose depends on a decision by the medical examiner to order postmortem toxicology, fatal overdoes may be under counted. ${ }^{22}, 23$ The inclusion of non-fatal overdose, identified using emergency medical services records, is also an important strength of our study, not present in many of the earlier studies.

Our results emphasize the importance of PIP as a contributing factor for fatal opioid overdose and can inform policies to address the problem of PIP. Public health campaigns are already underway to educate prescribers, patients, and the public, particularly regarding the danger from high-dose opioids and the importance of keeping first prescriptions low in dose and limited in duration. ${ }^{28}{ }^{29}$ A recent report by the Centers for Disease Control found that opioid prescribing peaked in 2010 and has declined since. ${ }^{30}$ Our study found 5 of 6 PIP indicators declining over time. However, co-prescribing benzodiazepines, which was strongly linked to overdose mortality in our study and has been the subject of an FDA Drug Safety Communication, ${ }^{31}$ increased over time and may be in need of a focused intervention. Our findings also suggest additional targets for improvement efforts, especially receipt of continuous opioids without a pain diagnosis. Prescribing without a pain diagnosis seems amenable to remediation-for example, through provider education, computer-based decision support, or indication-based prescribing. For prescribers, our results serve to emphasize an imperative to avoid PIP to the extent possible.

Our study also demonstrates how an online state prescription monitoring program can help identify PIP in real time. While previous studies have addressed this issue, ${ }^{32}, 33$ our study is the first to examine such a broad array of PIP subtypes simultaneously and to examine their independent effects in a single model. It might therefore be possible to derive and validate a clinical prediction rule or risk score, based on multiple PIP subtypes, that could potentially be displayed in real time for providers as part of the online state prescription monitoring program. Other options would include "flagging" evidence of PIP for providers. Such steps should be taken with care and rigorous evaluation.

Important issues remain. First, although it is logical to posit that efforts to reduce PIP will eventually lead to fewer adverse 
Table 3 Association of Potentially Inappropriate Opioid Prescribing (PIP) and the Outcomes of All-Cause Mortality, Fatal Opioid Overdose, and Non-fatal Opioid Overdose in Massachusetts from 2011 to $2015(n=3,078,034)$

\begin{tabular}{|c|c|c|c|c|c|c|}
\hline & \multicolumn{2}{|c|}{$\begin{array}{l}\text { All-cause mortality } \\
\text { AHR (95\% CI) }\end{array}$} & \multicolumn{2}{|c|}{$\begin{array}{l}\text { Fatal opioid overdose* } \\
\text { AHR (95\% CI) }\end{array}$} & \multicolumn{2}{|c|}{$\begin{array}{l}\text { Non-fatal opioid overdose }{ }^{\dagger} \\
\text { AHR }(95 \% \text { CI) }\end{array}$} \\
\hline & Model 1* & Model $2^{\S}$ & Model 1* & Model $2^{\S}$ & Model 1* & Model $2^{\S}$ \\
\hline$\geq 100$ MMEs $3+$ months & $3.85(3.78-3.92)$ & $2.18(2.14-2.23)$ & $6.31(5.74-6.94)$ & $2.22(1.99-2.48)$ & $2.13(2.03-2.23)$ & $1.68(1.59-1.76)$ \\
\hline Benzo overlap $3+$ months & $2.21(2.18-2.25)$ & $1.40(1.38-1.43)$ & $7.00(6.46-7.59)$ & $4.23(3.85-4.65)$ & $1.70(1.64-1.76)$ & $1.40(1.34-1.45)$ \\
\hline $4+$ prescribers & $2.59(2.54-2.64)$ & $1.37(1.34-1.40)$ & $3.55(3.23-3.91)$ & $1.32(1.18-1.48)$ & $1.45(1.39-1.51)$ & $1.01(0.96-1.06)$ \\
\hline 4+ pharmacies & $3.86(3.71-4.01)$ & $1.50(1.44-1.56)$ & $4.69(4.18-5.27)$ & $1.34(1.17-1.53)$ & $1.86(1.76-1.97)$ & $1.30(1.21-1.38)$ \\
\hline $3+$ cash payments & $1.99(1.96-2.02)$ & $1.30(1.27-1.32)$ & $2.80(2.49-3.15)$ & $1.03(0.91-1.17)$ & $1.32(1.25-1.39)$ & $0.96(0.90-1.01)$ \\
\hline No pain diagnosis & $2.90(2.85-2.96)$ & $2.05(2.01-2.09)$ & $5.95(5.26-6.72)$ & $2.74(2.40-3.13)$ & $2.60(2.38-2.84)$ & $2.21(2.02-2.41)$ \\
\hline
\end{tabular}

AHR adjusted hazard ratio, CI confidence interval, MME milligram morphine equivalents, PIP potentially inappropriate opioid prescribing

*Every fatal overdose, by definition, is also an all-cause mortality

${ }^{\dagger}$ For non-fatal overdose, patients are only considered to have been exposed to PIP if the exposure occurred before the outcome

${ }^{*}$ Model 1 is adjusted for age, sex, community-level poverty rate, depression, alcohol use disorder, rural town of residence, and the Elixhauser comorbidity index without the variables for depression and alcohol use disorder. Models for fatal outcomes (all-cause mortality and fatal opioid overdose) are also adjusted for history of incarceration and history of homelessness. For model 1, each cell represents the results of a separate regression analysis

${ }^{\xi}$ Model 2 is adjusted for the same covariates as model 1, plus the other PIP variables. For model 2, each column represents the results of a single regression analysis

events, there is a paucity of empirical studies regarding how long such decreases would take to occur. Second, it is unclear how best to manage patients who have already been exposed to PIP, although the findings of this study suggest that they would likely benefit from not being exposed to additional PIP subtypes. Further research to develop a more nuanced understanding of how PIP may contribute to negative outcomes,

Table 4 Association Between Potentially Inappropriate Opioid Prescribing (PIP) and the Outcomes of All-Cause Mortality, Fatal Opioid Overdose, and Non-fatal Opioid Overdose. Patients Are Characterized Based on How Many of the Six Types of PIP They Received $(n=3,078,034)$

\begin{tabular}{llll}
\hline \hline & $\begin{array}{l}\text { All-cause } \\
\text { mortality } \\
\text { AHR (95\% } \\
\text { CI)* }\end{array}$ & $\begin{array}{l}\text { Fatal opioid } \\
\text { overdose } \\
\text { AHR (95\% } \\
\text { CI)* }\end{array}$ & $\begin{array}{l}\text { Non-fatal opioid } \\
\text { overdose }^{*} \\
\text { AHR (95\% CI)* }\end{array}$ \\
\hline No PIP & REF & REF & REF \\
1 PIP & $\begin{array}{l}1.86 \\
(1.83-1.88)\end{array}$ & $4.24(3.86-4.66)$ & $1.54(1.47-1.60)$ \\
2 PIP & $\begin{array}{l}2.81 \\
(2.76-2.86)\end{array}$ & $7.05(6.32-7.86)$ & $1.98(1.89-2.08)$ \\
3 PIP & $\begin{array}{l}\text { (.10 } \\
(4.00-4.21)\end{array}$ & $\begin{array}{l}10.28(9.06- \\
11.67)\end{array}$ & $2.22(2.08-2.36)$ \\
4 or more & 5.37 & $12.99(11.22-$ & $2.18(2.02-2.36)$ \\
PIP & $(5.19-5.55)$ & $15.04)$ & \\
\hline
\end{tabular}

The six PIP variables are as follows: (1) $100 \mathrm{mg}$ morphine equivalents or greater on three or more occasions; (2) benzodiazepine/opioid overlap on three or more occasions; (3) four or more opioid prescribers in any one quarter; (4) four or more opioid pharmacies in any one quarter; (5) three or more cash payments; and (6) chronic opioids despite lack of a documented pain diagnosis. Patients with no PIP received opioids, but did not fulfill any of these criteria

AHR adjusted hazard ratio, CI confidence interval, MME milligram morphine equivalents, PIP potentially inappropriate opioid prescribing *Adjusted for age, sex, community-level poverty rate, depression, alcohol use disorder, rural town of residence, and the Elixhauser comorbidity index without the variables for depression and alcohol use disorder. Models for fatal outcomes (all-cause mortality and fatal opioid overdose) are also adjusted for history of incarceration and history of homelessness

${ }^{\dagger}$ Every fatal overdose, by definition, is also an all-cause mortality

${ }^{7}$ For non-fatal overdose, patients are only considered to have been exposed to PIP if the exposure occurred before the outcome within what time frame, under what circumstances, and for which patients, is needed.

Our study has noteworthy strengths. To identify patients who received an opioid prescription, we used the online state prescription monitoring program, which identified all outpatient pharmacy-dispensed opioid prescriptions in the state. An additional strength is our examination of six types of PIP, some of which have not been examined previously.

We also acknowledge the study's limitations. First, while we controlled for relevant covariates such as age and burden of comorbidity, as with any observational study, we were unable to control for all relevant patient-level factors, as some are not routinely measured. Second, the reasons why patients received opioids can be unclear in a large, automated dataset such as the one we used - especially when a patient has more than one pain condition over time. Therefore, we were not able to control for the reason patients received opioids, or their level of pain. Third, because the dataset began in 2011, some of the patients who appeared to receive their first opioid prescription in that year may have received one earlier, and we have no way to account for this.

Fourth, the population-based results from Massachusetts may not fully generalize to other states, nor to minors or patients with cancer. PIP and its consequences may look somewhat different in other populations. Fifth, we also only examined the first non-fatal overdose observed and do not know to what extent our findings would apply to multiple non-fatal overdoses. Sixth, while this dataset featured extremely strong outcomes ascertainment for a study of this type, there is still a known issue with the underascertainment of fatal opioid overdose, as has been explored in several recent studies. ${ }^{22}, 23$ Like any study of this kind, our analyses of fatal opioid overdose were limited to those instances that were appropriately recognized and coded. This, in part, was the rationale for including allcause mortality as a study outcome. 
Seventh, while our study examined a broader range of PIP subtypes than previous studies, there are other potential indicators of PIP such as long-acting as opposed to short-acting opioids, ${ }^{34}, 35$ overlapping opioid prescriptions, ${ }^{4}, 14,18$ and prescribing opioid analgesics to patients with a history of substance misuse ${ }^{14}$ that have been linked with opioid overdose, and we do not know how the inclusion of these additional PIPs would have influenced our findings.

Eighth, at least one of our PIP variables has not been examined in previous studies: receiving chronic opioid therapy without a documented pain diagnosis. We believe this variable is reasonable, since it seems unlikely that a pain condition of sufficient severity to require long-term opioid therapy would not generate any billing codes over a fiveyear period, even for a patient with a relatively stable pain diagnosis. Nevertheless, we recognize that there may be situations in which clinicians are appropriately prescribing opioids without recording such a diagnosis. In addition, while we based our list of pain diagnoses on a list of commonly submitted codes from pain clinics, it is possible that including different pain diagnoses would have changed this variable slightly. However, given the strong effect of this variable, minor changes in its composition would be unlikely to strongly alter the results.

Ninth, our dataset did not capture prescriptions from military facilities, and data from Veterans Health Administration facilities only began to be captured in 2014. Tenth, this dataset did not allow us to identify individual prescribers, preventing us from ascertaining whether PIP is concentrated among a small group of prescribers. Finally, the limited time period available to complete our analysis limited our ability to conduct more sophisticated analyses, such as examining the temporal relationships between exposure and outcomes, or the various steps along the pathway from PIP to death, such as the transition to misuse of prescribed opioids. The Massachusetts legislature has recently determined that Chapter 55 data will be made available for longer term studies, and such analyses are needed to help formulate more effective intervention approaches.

Despite these limitations, our findings regarding the relationship of PIP with fatal overdose, non-fatal overdose, and all-cause mortality suggest that while any opioid prescription may carry some risk, we can identify a subset of prescriptions that are particularly risky. The ability to identify PIP using a state-level online prescription monitoring program, which all states now have, may provide an opportunity to intervene in real time to reduce PIP and to protect patients who have received PIP. The urgency of the opioid crisis necessitates an aggressive response, and the strategy discussed here seems a promising step forward, together with appropriate evaluation of its real-world impact.

Corresponding Author: Adam J. Rose, MD MSc FACP; RAND Corporation, Boston, MA, USA (e-mail: arose@rand.org).
Funding This study was funded by the GE Foundation (PI: Stopka). The funder had no role in the design, conduct, or reporting of the study.

Compliance with Ethical Standards:

Prior Presentations: None

Conflict of Interest: The authors declare that they do not have a conflict of interest.

Disclaimer: The opinions expressed in this manuscript are those of the authors and do not represent the official views or policies of the Massachusetts Department of Public Health or the Commonwealth of Massachusetts.

Responsible Research Statement: Study protocol and statistical code: available from Dr. Rose (email, arose@rand.org). Dataset: those interested in using the data may contact Dana Bernson (email, dana.bernson@state.ma.us). All interested parties are encouraged to apply; however, permission to use the data will be prioritized in accordance with current Massachusetts Department of Public Health priorities.

\section{REFERENCES}

1. Rudd RA, Seth P, David F, Scholl L. Increases in drug and opioidinvolved overdose deaths-United States, 2010-2015. MMWR Morb Mortal Wkly Rep. 2016;65:1445-52.

2. Schuchat A, Houry D, Guy GP. New data on opioid use and prescribing in the United States. JAMA. 2017;318:425-426.

3. Jones CM, Paulozzi LJ, Mack KA. Sources of prescription opioid pain relievers by frequency of past-year nonmedical use United States, 20082011. JAMA Intern Med. 2014;174:802-3.

4. Liu Y, Logan JE, Paulozzi LJ, Zhang K, Jones CM. Potential misuse and inappropriate prescription practices involving opioid analgesics. Am J Manag Care. 2013;19:648-665.

5. Paulozzi LJ, Strickler GK, Kreiner PW, Koris CM. Controlled substance prescribing patterns-prescription behavior surveillance system, eight states, 2013. MMWR Surveill Summ. 2015;64(SS09):1-14.

6. Gellad WF, Zhao X, Thorpe CT, et al. Overlapping buprenorphine, opioid, and benzodiazepine prescriptions among veterans dually enrolled in Department of Veterans Affairs and Medicare Part D. Subst Abus. 2017;38:22-5.

7. Gwira Baumblatt JA, Wiedeman C, et al. High-risk use by patients prescribed opioids for pain and its role in overdose deaths. JAMA Intern Med. 2014;174:796-801.

8. Bohnert AS, Logan JE, Ganoczy D, Dowell D. A detailed exploration into the association of prescribed opioid dosage and overdose deaths among patients with chronic pain. Med Care. 2016;54:435-41.

9. Bohnert AS, Valenstein M, Bair MJ, et al. Association between opioid prescribing patterns and opioid overdose-related deaths. JAMA. 2011;305:1315-21.

10. Dunn KM, Saunders KW, Rutter CM, et al. Opioid prescriptions for chronic pain and overdose: a cohort study. Ann Intern Med. 2010; 152:85-92.

11. Zedler B, Xie L, Wang $\mathbf{L}$, et al. Risk factors for serious prescription opioid-related toxicity or overdose among Veterans Health Administration patients. Pain Med. 2014;15:1911-29.

12. Gomes T, Mamdani MM, Dhalla IA, Paterson JM, Juurlink DN. Opioid dose and drug-related mortality in patients with nonmalignant pain. Arch Intern Med. 2011;171:686-91.

13. Garg RK, Fulton-Kehoe D, Franklin GM. Patterns of opioid use and risk of opioid overdose death among medicaid patients. Med Care. 2017;55:661-8.

14. Cochran G, Gordon AJ, Lo-Ciganic WH, et al. An examination of claims-based predictors of overdose from a large medicaid program. Med Care. 2017;55:291-8.

15. Dasgupta N, Funk MJ, Proescholdbell S, Hirsch A, Ribisl KM, Marshall S. Cohort study of the impact of high-dose opioid analgesics on overdose mortality. Pain Med. 2016;17:85-98.

16. Park TW, Saitz R, Ganoczy D, Ilgen MA, Bohnert AS. Benzodiazepine prescribing patterns and deaths from drug overdose among US veterans receiving opioid analgesics: case-cohort study. BMJ. 2015;350:h2698. 
17. Hall AJ, Logan JE, Toblin RL, et al. Patterns of abuse among unintentional pharmaceutical overdose fatalities. JAMA. 2008;300:2613-20.

18. Yang Z, Wilsey B, Bohm M, et al. Defining risk of prescription opioid overdose: pharmacy shopping and overlapping prescriptions among longterm opioid users in medicaid. J Pain. 2015; 16:445-53.

19. Commonwealth of Massachusetts: Chapter 55 Website. Accessed at http://www.mass.gov/eohhs/gov/departments/dph/stop-addiction/ chapter-55-overdose-assessment.html on May 22, 2018.

20. Chapter 55: An Act Requiring Certain Reports for Opiate Overdoses Initial Report. Accessed at http://www.mass.gov/eohhs/docs/dph/stop addiction/dph-legislative-report-chapter-55-opioid-overdose-study-9-15 2016.pdf on May 22, 2018.

21. Report to the Massachuetts Legislature. "An Assessment of Fatal and Nonfatal Opioid Overdoses in Massachusetts (2011-2015)." Available at: https://www.mass.gov/files/documents/2017/08/31/legislative-report-chapter-55-aug-2017.pdf. Accessed May 22, 2018.

22. Ruhm CJ. Geographic variation in opioid and heroin involved drug poisioning mortality rates. Am J Prev Med. 2017;53:745-53.

23. Rockett IR, Hobbs GR, Wu D, et al. Variable classification of drug intoxication suicides across US states: a partial artifact of forensics? PLoS One. 2015;10:e135296.

24. United States Census Bureau. American FactFinder. Accessed at http:// factfinder.census.gov/ on May 22, 2018.

25. Krieger N, Chen JT, Waterman PD, Rehkopf DH, Subramanian SV. Painting a truer picture of US socioeconomic and racial/ethnic health inequalities: the Public Health Disparities Geocoding Project. Am J Public Health. 2005;95:312-23.
26. Quan $\mathbf{H}$, Sundararajan V, Halfon $\mathbf{P}$, et al. Coding algorithms for defining comorbidities in ICD-9-CM and ICD-10 administrative data. Med Care. 2005;43:1130-9.

27. VanderWeele TJ, Ding $\mathbf{P}$. Sensitivity analysis in observational research: introducing the E-value. Ann Intern Med. 2017;167:268-74.

28. Dowell D, Haegerich TM, Chou R. CDC Guideline for prescribing opioids for chronic pain-United States, 2016. JAMA. 2016;315:1624-45.

29. Massachusetts Medical Society. Smart and safe: information about opioid medications for patients, prescribers, and policymakers. Accessed at http://www.massmed.org/smartandsafe/ on May 22, 2018.

30. Guy GP, Zhang $\mathbf{K}$, Bohm MK, et al. Vital signs: changes in opioid prescribing in the United States, 2006-2015. MMWR Morb Mortal Wkly Rep. 2017;66:697-704.

31. US Food and Drug Administration. FDA Drug Safety Communication: FDA warns about serious risks and death when combining opioid pain or cough medicines with benzodiazepines; requires its strongest warning. Available at: https://www.fda.gov/Drugs/DrugSafety/ucm518473.htm. Accessed May 22, 2018.

32. Katz N, Panas L, Kim M, et al. Usefulness of prescription monitoring programs for surveillance-analysis of Schedule II opioid prescription data in Massachusetts, 1996-2006. Pharmacoepidemiol Drug Saf 2010;19:115-23.

33. Pardo B. Do more robust prescription drug monitoring programs reduce prescription opioid overdose? Addiction. 2016;112:1773-83.

34. Miller M, Barber CW, Leatherman S, et al. Prescription opioid duration of action and the risk of unintentional overdose among patients receiving opioid therapy. JAMA Intern Med. 2015;175:608-15.

35. Ray WA, Chung CP, Murray KT, Hall K, Stein CM. Prescription of longacting opioids and mortality in patients with chronic noncancer pain. JAMA. 2016;315:2415-23. 